PROCEEDINGS OF THE

AMERICAN MATHEMATICAL SOCIETY

Volume 137, Number 12, December 2009, Pages 4227-4242

S 0002-9939(09)10047-3

Article electronically published on August 4, 2009

\title{
A UNIFORM ESTIMATE FOR FOURIER RESTRICTION TO SIMPLE CURVES
}

DANIEL M. OBERLIN

(Communicated by Michael T. Lacey)

\begin{abstract}
We prove a uniform Fourier extension-restriction estimate for a certain class of curves in $\mathbb{R}^{d}$.
\end{abstract}

\section{INTRODUCTION}

Let $\gamma$ be a curve in $\mathbb{R}^{d}$ given by

$$
\gamma(t)=\left(t, \frac{t^{2}}{2}, \ldots, \frac{t^{d-1}}{(d-1) !}, \phi(t)\right)
$$

where $\phi \in C^{(d+2)}(a, b)$ and $\phi^{(j)}(t)>0$ for $t \in(a, b), j=0,1,2, \ldots, d+2$. Such curves are termed simple in [5]. Write $\omega(t)$ for $\phi^{(d)}(t)$. The purpose of this note is to prove a uniform Fourier extension-restriction theorem for affine arclength measure on curves (1.1):

Theorem 1.1. Suppose $\lambda$ is the measure on $\gamma$ given by $d \lambda=\omega(t)^{2 /\left(d^{2}+d\right)} d t$. If $1 \leq p<d+2$ and $\frac{1}{p}+\frac{d(d+1)}{2} \frac{1}{q}=1$, then there is $C(p, d)$ such that the following estimate holds:

$$
\|\widehat{f d \lambda}\|_{q} \leq C(p, d)\|f\|_{L^{p}(\lambda)} .
$$

If $d=2$ this is just the theorem in [7], a result originally established in slightly more generality, but with a more complicated proof, by Sjölin in [9]. This note is the result of an attempt to apply the method from [7] in higher dimensions. Theorem 1.1. which is an immediate consequence of Theorems 1.2 and 1.3 below, is somewhat analogous to the result of [4] on two fronts: it is a direct consequence of a geometric inequality (Theorem 1.2 here) combined with a fairly simple argument (Theorem 1.3 here), and its range of exponents $p$ is the (probably suboptimal) range obtained by Christ [3. For a better range of $p$ when $\omega$ satisfies a certain auxiliary condition, see Theorem 1.1 in [2]. For some of the history of the problem of restricting Fourier transforms to curves, see 1 .

Received by the editors November 6, 2008, and, in revised form, May 26, 2009.

2000 Mathematics Subject Classification. Primary 42B10.

Key words and phrases. Fourier transforms of measures on curves, Fourier restriction problem, affine arclength measure.

The author was supported in part by NSF grant DMS-0552041.

(C)2009 American Mathematical Society Reverts to public domain 28 years from publication 
Theorem 1.2. There exists $C=C(d)$ such that the estimate

$$
\begin{aligned}
\int_{a}^{b}\left(\int_{\left\{t_{1} \leq t_{i}<b\right\}} \chi_{F}\left(\gamma\left(t_{1}\right)+\cdots+\gamma\left(t_{d}\right)\right)\left[\prod_{i=2}^{d} \omega\left(t_{i}\right)\right]^{2 /\left(d^{2}+d\right)} d t_{2} \cdots d t_{d}\right)^{(d+2) / 2} & \\
& \times \omega\left(t_{1}\right)^{2 /\left(d^{2}+d\right)} d t_{1} \leq C(d) m_{d}(F)
\end{aligned}
$$

holds for Borel $F \subset \mathbb{R}^{d}$.

The next result is Theorem 7 in 8$]$.

Theorem 1.3. Suppose that $\lambda$ is a nonnegative Borel measure on $\mathbb{R}^{d}$ satisfying the inequality

$$
\int\left(\int_{\left\{\tau\left(y_{1}\right) \leq \tau\left(y_{i}\right)\right\}} \chi_{F}\left(y_{1}+y_{2}+\cdots+y_{m}\right) d \lambda\left(y_{2}\right) \cdots d \lambda\left(y_{m}\right)\right)^{\frac{m+2}{2}} d \lambda\left(y_{1}\right) \leq c m_{d}(F)
$$

for some nonnegative integer $m \geq 3$, some real-valued Borel function $\tau$ on supp $(\lambda)$, and all Borel $F \subseteq \mathbb{R}^{d}$. Then the adjoint restriction estimate

$$
\|\widehat{f d \lambda}\|_{q} \leq C(c, p)\|f\|_{L^{p}(\lambda)}
$$

holds whenever $\frac{1}{p}+\frac{m(m+1)}{2} \frac{1}{q}=1$ and $1 \leq p<m+2$.

The next section contains the proof of Theorem 1.2, while $\S 3$ contains the proofs of certain lemmas used in $\S 2$.

\section{Proof of Theorem 1.2}

The idea for proving Theorem [1.2, from $\left[8\right.$, is to regard (1.2) as an $L^{(d+2) / 2,1} \rightarrow$ $L^{(d+2) / 2}$ estimate for a certain operator $T$ and to establish $(1.2)$ by establishing the dual $L^{(d+2) / d} \rightarrow L^{(d+2) / d, \infty}$ estimate for $T^{*}$. Let $J\left(t_{1}, \ldots, t_{d}\right)$ be the absolute value of the Jacobian determinant for the map

$$
\left(t_{1}, \ldots t_{d}\right) \mapsto \gamma\left(t_{1}\right)+\cdots+\gamma\left(t_{d}\right) .
$$

Then

$$
\begin{aligned}
& \left\langle f, T^{*} g\right\rangle=\langle T f, g\rangle \\
& =\int_{a}^{b}\left(\int_{\left\{t_{1} \leq t_{i}<b\right\}} f\left(\gamma\left(t_{1}\right)+\cdots+\gamma\left(t_{d}\right)\right)\left[\prod_{i=2}^{d} \omega\left(t_{i}\right)\right]^{2 /\left(d^{2}+d\right)} d t_{2} \cdots d t_{d}\right) \\
& \times g\left(t_{1}\right) \omega\left(t_{1}\right)^{2 /\left(d^{2}+d\right)} d t_{1} \\
& =\int_{\left\{a<t_{1} \leq t_{i}<b\right\}}\left(\frac{g\left(t_{1}\right)}{J\left(t_{1}, \ldots, t_{d}\right)}\left[\prod_{i=1}^{d} \omega\left(t_{i}\right)\right]^{2 /\left(d^{2}+d\right)}\right) f\left(\gamma\left(t_{1}\right)+\cdots+\gamma\left(t_{d}\right)\right) \\
& \times J\left(t_{1}, \ldots, t_{d}\right) d t_{d} \cdots d t_{1}
\end{aligned}
$$

so that the desired $L^{(d+2) / d} \rightarrow L^{(d+2) / d, \infty}$ estimate for nonnegative $g$,

$$
\lambda^{(d+2) / d} m_{d}\left\{T^{*} g \geq \lambda\right\} \leq C(d) \int_{a}^{b} g\left(t_{1}\right)^{(d+2) / d} \omega\left(t_{1}\right)^{2 /\left(d^{2}+d\right)} d t_{1} \quad(\lambda>0),
$$


will follow from the estimate

$$
\begin{aligned}
\lambda^{(d+2) / d} \int \chi_{\widetilde{E}}\left(t_{1}, \ldots, t_{d}\right) J\left(t_{1}, \ldots, t_{d}\right) d t_{d} & \cdots d t_{1} \\
& \leq C(d) \int_{a}^{b} g\left(t_{1}\right)^{(d+2) / d} \omega\left(t_{1}\right)^{2 /\left(d^{2}+d\right)} d t_{1},
\end{aligned}
$$

where

$$
\widetilde{E}=\left\{\left(t_{1}, \ldots, t_{d}\right): a<t_{1} \leq \cdots \leq t_{d}<b, \frac{g\left(t_{1}\right)}{J\left(t_{1}, \ldots, t_{d}\right)}\left[\prod_{i=1}^{d} \omega\left(t_{i}\right)\right]^{2 /\left(d^{2}+d\right)} \geq \lambda\right\} .
$$

(The change of variables implicit in this argument can be justified as in [6], p. 549.) By absorbing $\lambda$ into $g$ we can assume $\lambda=1$. Thus (2.1) will follow from integrating the inequality

$$
\int \chi_{\widetilde{E}}\left(t_{1}, \ldots, t_{d}\right) J\left(t_{1}, \ldots, t_{d}\right) d t_{d} \cdots d t_{2} \leq C(d) g\left(t_{1}\right)^{(d+2) / d} \omega\left(t_{1}\right)^{2 /\left(d^{2}+d\right)}
$$

with respect to $t_{1}$. Lemma 2.3 in 2 shows that there is a nonnegative function $\psi=\psi\left(u ; t_{1}, \ldots, t_{d}\right)$ supported in $\left[t_{1}, t_{d}\right]$ such that

$$
J\left(t_{1}, \ldots, t_{d}\right)=\int_{t_{1}}^{t_{d}} \omega(u) \psi\left(u ; t_{1}, \ldots, t_{d}\right) d u
$$

and so (2.2) will follow from the inequality

$$
\int \chi_{\widetilde{E}}\left(t_{1}, \ldots, t_{d}\right) J\left(t_{1}, \ldots, t_{d}\right) d t_{d} \cdots d t_{2} \leq C(d) c^{(d+2) / d} \omega\left(t_{1}\right)^{2 /\left(d^{2}+d\right)},
$$

to hold for $c>0$ and $t_{1} \in(a, b)$, where now

$$
\begin{aligned}
\widetilde{E}=\left\{\left(t_{1}, \ldots, t_{d}\right): a<t_{1} \leq\right. & \ldots t_{d}<b, \\
& \left.\int_{t_{1}}^{t_{d}} \omega(u) \psi\left(u ; t_{1}, \ldots, t_{d}\right) d u \leq c\left[\prod_{i=1}^{d} \omega\left(t_{i}\right)\right]^{2 /\left(d^{2}+d\right)}\right\} .
\end{aligned}
$$

Homogeneity allows absorbing $c$ into $\omega$, so we can assume $c=1$. With $t_{1}>a$ fixed, then, and with

$$
E=\left\{\left(t_{2}, \ldots, t_{d}\right): t_{1} \leq t_{2} \leq \cdots \leq t_{d}<b, J\left(t_{1}, \ldots, t_{d}\right) \leq\left[\prod_{i=1}^{d} \omega\left(t_{i}\right)\right]^{2 /\left(d^{2}+d\right)}\right\},
$$

inequality (2.4), and so (1.2), will follow from

$$
\int \chi_{E}\left(t_{2}, \ldots, t_{d}\right)\left[\prod_{i=2}^{d} \omega\left(t_{i}\right)\right]^{2 /\left(d^{2}+d\right)} d t_{d} \cdots d t_{2} \leq C(d) .
$$

To begin the proof of (2.6), let $J \subset \mathbb{Z}$ be an interval of integers such that $\left\{2^{j}: j \in J\right\}$ is the set of dyadic values assumed by $\omega$ on $(a, b)$. For each $j \in J$, choose $a_{j} \in(a, b)$ such that $\omega\left(a_{j}\right)=2^{j}$. If $J$ has a least element, say $j_{\min }$, we let $a_{j_{\min }-1}=a$ and append $j_{\min }-1$ to $J$. If $J$ has a greatest element, we make a similar accommodation. Then, writing $I_{j}=\left[a_{j}, a_{j+1}\right) \cap(a, b)$, we obtain a partition $\left\{I_{j}\right\}_{j \in J}$ of $(a, b)$. 
The proof of (2.6) simplifies significantly in the case $d=2$. For the sake of the reader who would see the main idea of the proof but be spared its unfortunately complicated notation, we will first sketch the proof in that special case. So assume, for the moment, that $d=2$. Since $\psi\left(u ; t_{1}, t_{2}\right)=\chi_{\left(t_{1}, t_{2}\right)}(u)$, (2.3) shows that (2.5) becomes

$$
E=\left\{t_{2}: t_{2} \geq t_{1}, \int_{t_{1}}^{t_{2}} \omega(u) d u \leq\left[\omega\left(t_{1}\right) \omega\left(t_{2}\right)\right]^{1 / 3}\right\}
$$

while (2.6) becomes

$$
\int \chi_{E}\left(t_{2}\right) \omega\left(t_{2}\right)^{1 / 3} d t_{2} \leq C
$$

Let $E_{j}=E \cap I_{j}$ and $s_{j}=\sup E_{j}$. Then, with $j_{1}$ chosen so that $t_{1} \in I_{j_{1}}$, there are the inequalities

$$
\int_{E_{j}} \omega(u) d u \leq \int_{t_{1}}^{s_{j}} \omega(u) d u \leq\left[\omega\left(t_{1}\right) \omega\left(s_{j}\right)\right]^{1 / 3} \lesssim\left(2^{j_{1}} 2^{j}\right)^{1 / 3}
$$

(by (2.7) and the definitions of $s_{j}$ and $E_{j}$ ). Thus

$$
\int_{E_{j}} \omega(u)^{1 / 3} d u \sim 2^{-2 j / 3} \int_{E_{j}} \omega(u) d u \lesssim\left(2^{j_{1}} 2^{-j}\right)^{1 / 3} .
$$

Since $j \geq j_{1}$, (2.8) now follows by summing a geometric series.

We return now to the proof of the general case of (2.6). With $t_{1} \in(a, b)$ fixed, say $t_{1} \in I_{j_{1}}$, with $E$ as in (2.5), and for integers $j_{2} \leq j_{3} \leq \cdots \leq j_{d}$ in $J$ with $j_{2} \geq j_{1}$, we set

$$
E_{j_{2} \cdots j_{d}}=\left\{\left(t_{2}, \ldots, t_{d}\right) \in E:\left(t_{2}, \ldots, t_{d}\right) \in I_{j_{2}} \times \cdots \times I_{j_{d}}\right\} .
$$

The desired estimate (2.6) will follow from

$$
\sum_{j_{2} \geq j_{1}} \cdots \sum_{j_{d} \geq j_{d-1}}\left(2^{j_{2}+\cdots+j_{d}}\right)^{2 /\left(d^{2}+d\right)} m_{d-1}\left(E_{j_{2} \cdots j_{d}}\right) \leq C(d) .
$$

To establish (2.9) it is enough to show that, for each $\left(j_{2}, \ldots, j_{d}\right)$ figuring in the sum in (2.9), we have

$$
\left(2^{j_{2}+\cdots+j_{d}}\right)^{1 /(d-1)} m_{d-1}\left(E_{j_{2} \cdots j_{d}}\right)^{d / 2} \leq C(d)\left(2^{j_{1}+j_{2}+\cdots+j_{d}}\right)^{2 /\left(d^{2}+d\right)} .
$$

In fact, some algebra shows that (2.10) is equivalent to

$$
\left(2^{j_{2}+\cdots+j_{d}}\right)^{2 /\left(d^{2}+d\right)} m_{d-1}\left(E_{j_{2} \cdots j_{d}}\right) \leq C(d) 2^{4 j_{1} /\left(d^{3}+d^{2}\right)} 2^{-4\left(j_{2}+\cdots+j_{d}\right) /\left[\left(d^{3}+d^{2}\right)(d-1)\right]}
$$

and so, given (2.10), (2.9) follows by summing a geometric series.

Moving towards the proof of (2.10), fix $\left(j_{2}, \ldots, j_{d}\right)$. In what follows we will often write $j(l)$ instead of $j_{l}$. Let $p_{1}<p_{2}<\cdots p_{k-1}$ be the indices $i$ in $\{1,2, \ldots, d-1\}$ for which $j(i+1)-j(i) \geq 2$ and set $p_{0}=0$ and $p_{k}=d$. Define $\ell_{1}, \ldots, \ell_{k}$ by $\ell_{n}=p_{n}-p_{n-1}-1(n=1, \ldots, k)$, and observe that $\ell_{1}+\cdots+\ell_{k}=d-k$. Then

$$
\begin{gathered}
\{j(1), j(2), \ldots, j(d)\} \\
=\left\{j\left(p_{0}+1\right), j\left(p_{0}+2\right), \ldots, j\left(p_{1}\right) ; j\left(p_{1}+1\right), \ldots, j\left(p_{2}\right) ; \ldots ; j\left(p_{k-1}+1\right), \ldots, j\left(p_{k}\right)\right\}
\end{gathered}
$$

where if $j(i)$ and $j(i+1)$ are separated by a semicolon, then $j(i+1)-j(i) \geq 2$, and otherwise $0 \leq j(i+1)-j(i) \leq 1$. Next we construct $k$ subintervals $J_{n}$ of $(a, b)$ by setting, for $n=1, \ldots, k$,

$$
J_{n}=I_{j\left(p_{n-1}+1\right)} \cup I_{j\left(p_{n-1}+2\right)} \cup \cdots \cup I_{j\left(p_{n}\right)}
$$


so that, recalling the definition of $I_{j}$, the endpoints $c_{n}$ and $d_{n}$ of $J_{n}$ are given by $c_{n}=a_{j\left(p_{n-1}+1\right)}$ and $d_{n}=a_{j\left(p_{n}\right)+1}$. Note that $c_{1}<d_{1}<c_{2}<d_{2}<\cdots<c_{k}<d_{k}$ (see (2.13) below) and that if $\left(t_{2}, \ldots, t_{d}\right) \in E_{j_{2} \ldots j_{d}}$, then

$$
c_{n} \leq t_{p_{n-1}+1} \leq t_{p_{n-1}+2} \leq \cdots \leq t_{p_{n}} \leq d_{n} .
$$

We will need the facts that if $n=2, \ldots k$, then

$$
d_{n-1} \leq a_{j\left(p_{n-1}+1\right)-1}<c_{n}
$$

and

$$
c_{n}-a_{j\left(p_{n-1}+1\right)-1} \gtrsim d_{n}-c_{n} .
$$

(Throughout this note, the constants implied by symbols such as $\lesssim$ can easily be checked to depend only on $d$.) To see (2.14), note that because $\omega\left(a_{j}\right)=2^{j}$ and $\omega^{\prime}$ is nondecreasing we have

$$
\left(a_{j+1}-a_{j}\right) \omega^{\prime}\left(a_{j}\right) \leq \int_{a_{j}}^{a_{j+1}} \omega^{\prime}(u) d u=2^{j}=2 \int_{a_{j-1}}^{a_{j}} \omega^{\prime}(u) d u \leq 2\left(a_{j}-a_{j-1}\right) \omega^{\prime}\left(a_{j}\right)
$$

so that $\left(a_{j+1}-a_{j}\right) \leq 2\left(a_{j}-a_{j-1}\right)$ and therefore

$$
\left(a_{j+p}-a_{j+p-1}\right) \leq 2^{p}\left(a_{j}-a_{j-1}\right) .
$$

Now, by definition of $p_{n-1}, j\left(p_{n-1}\right)+1 \leq j\left(p_{n-1}+1\right)-1$, and so $a_{j\left(p_{n-1}+1\right)-1}$ lies between $d_{n-1}=a_{j\left(p_{n-1}\right)+1}$ and $c_{n}=a_{j\left(p_{n-1}+1\right)}$ in the sense of (2.13). Also, according to (2.11),$J_{n}=\left(c_{n}, d_{n}\right)$ is (up to endpoints) the union of no more than $d$ intervals $I_{j}=\left[a_{j}, a_{j+1}\right)$. By choice of the $p_{n}$, each interval but the first in the union in (2.11) is either identical to or contiguous to the one on its left. Since the first of these intervals is $\left(a_{j\left(p_{n-1}+1\right)}, a_{j\left(p_{n-1}+1\right)+1}\right)$, (2.15) implies (2.14).

We now outline the proof of (2.10), beginning with a lemma (the proofs of the lemmas will be given in 93 :

Lemma 2.1. Suppose $t_{1}<\cdots<t_{d}$ and $\left(\alpha_{i}, \beta_{i}\right) \subset\left(t_{i}, t_{i+1}\right)$. Write $\Delta_{i}=\beta_{i}-\alpha_{i}$ and suppose

$$
f=\sum_{i=1}^{d-1} \nu_{i} \chi_{\left(\alpha_{i}, \beta_{i}\right)}
$$

where $\nu_{i} \geq 0$. Fix $p \in\{1, \ldots, d-1\}$. Suppose

$$
\left\{e_{i}: i=1,2, \ldots d-1, i \neq p\right\}=\{1,2, \ldots, d-2\} .
$$

Then

$$
\int_{t_{1}}^{t_{d}} f(u) \psi\left(u ; t_{1}, \ldots, t_{d}\right) d u \gtrsim \nu_{p} \Delta_{p}^{d-1} \prod_{\substack{1 \leq i \leq d-1 \\ i \neq p}} \Delta_{i}^{e_{i}} .
$$

With $t_{1}$ and $j_{2}, \ldots, j_{d}$ fixed and with $\left(t_{2}, \ldots, t_{d}\right) \in E_{j_{2} \ldots j_{d}}$, we will apply Lemma 2.1 to a collection $\mathcal{I}$ of intervals $\left(\alpha_{i}, \beta_{i}\right)$ specified as follows: for $n=1, \ldots, k$ and $i=p_{n-1}+1, \ldots, p_{n}-1$, the $\ell_{n}$ intervals $\left(t_{i}, t_{i+1}\right)$ will be in $\mathcal{I}$; additionally, for $n=2, \ldots, k$, the intervals $\tilde{J}_{n}$ defined by

$$
\tilde{J}_{n}=\left(a_{j\left(p_{n-1}+1\right)-1}, c_{n}\right) \subset\left(t_{p_{n-1}}, t_{p_{n-1}+1}\right)
$$

will be in $\mathcal{I}$ (we set $\widetilde{J}_{1}=\emptyset$ ). Observe that there are integers $m_{1} \leq m_{2} \leq \cdots \leq m_{k}$ such that if

$$
\mathcal{J}_{n}=\tilde{J}_{n} \cup\left(\bigcup_{i=p_{n-1}+1}^{p_{n}-1}\left(t_{i}, t_{i+1}\right)\right),
$$


then

$$
\omega \sim 2^{m_{n}} \text { on } \mathcal{J}_{n}(n=1, \ldots, k) .
$$

(This is true because, according to (2.12), $\mathcal{J}_{n}$ is contained in the union of at most $\ell_{n}+1 \leq d$ contiguous intervals $I_{j}$, and $\omega \sim 2^{j}$ on $I_{j}$.) Then (2.10) can be written as

$$
\begin{array}{r}
2^{\left[\ell_{1} m_{1}+\left(\ell_{2}+1\right) m_{2}+\cdots+\left(\ell_{k}+1\right) m_{k}\right] /(d-1)} m_{d-1}\left(E_{j_{2} \cdots j_{d}}\right)^{d / 2} \\
\lesssim 2^{2\left[\left(\ell_{1}+1\right) m_{1}+\left(\ell_{2}+1\right) m_{2}+\cdots+\left(\ell_{k}+1\right) m_{k}\right] /\left(d^{2}+d\right)} .
\end{array}
$$

Similarly, the inequality

$$
J\left(t_{1}, \ldots, t_{d}\right) \leq\left[\prod_{i=1}^{d} \omega\left(t_{i}\right)\right]^{2 /\left(d^{2}+d\right)}
$$

in (2.5) implies that

$$
J\left(t_{1}, \ldots, t_{d}\right) \lesssim 2^{2\left[\left(\ell_{1}+1\right) m_{1}+\cdots+\left(\ell_{k}+1\right) m_{k}\right] /\left(d^{2}+d\right)} .
$$

Now, as we will see below, Lemma 2.1, (2.17), (2.19), and

$$
J\left(t_{1}, \ldots, t_{d}\right)=\int_{t_{1}}^{t_{d}} \omega(u) \psi\left(u ; t_{1}, \ldots, t_{d}\right) d u
$$

will yield certain estimates of the form

$$
2^{m_{n}} \Delta_{p}^{d-1} \prod_{\substack{1 \leq i \leq d-1 \\ i \neq p}} \Delta_{i}^{e_{i}} \lesssim 2^{2\left[\left(\ell_{1}+1\right) m_{1}+\left(\ell_{2}+1\right) m_{2}+\cdots+\left(\ell_{k}+1\right) m_{k}\right] /\left(d^{2}+d\right)}
$$

when $\left(\alpha_{p}, \beta_{p}\right) \subset \mathcal{J}_{n}$. A weighted geometric mean of these estimates (2.20) will give

$$
\begin{aligned}
2^{\left[\ell_{1} m_{1}+\left(\ell_{2}+1\right) m_{2}+\cdots+\left(\ell_{k}+1\right) m_{k}\right] /(d-1)} & W_{1}^{d /\left(\ell_{1}+1\right)} \prod_{n=2}^{k}\left(\rho_{n}^{\left(d+\ell_{n}\right) / 2} W_{n}^{(d-1) /\left(\ell_{n}+1\right)}\right) \\
& \lesssim 2^{2\left[\left(\ell_{1}+1\right) m_{1}+\left(\ell_{2}+1\right) m_{2}+\cdots+\left(\ell_{k}+1\right) m_{k}\right] /\left(d^{2}+d\right)},
\end{aligned}
$$

where $\rho_{n}$ is the length of $\tilde{J}_{n}$ and with the $W_{n}$ 's given by

$$
W_{n}=W\left(t_{p_{n-1}+1}, \ldots, t_{p_{n}}\right)
$$

where, for $s_{1} \leq \cdots \leq s_{m}$,

$$
W\left(s_{1}, \ldots, s_{m}\right)=\sup \left\{\prod_{i=1}^{m-1}\left(s_{i+1}-s_{i}\right)^{e_{i}}:\left\{e_{1}, \ldots, e_{m-1}\right\}=\{1, \ldots, m-1\}\right\} .
$$

Lemma 2.3 below will allow the choice of $\left(t_{2}, \ldots, t_{d}\right) \in E_{j_{2} \ldots j_{d}}$ such that

$$
m_{d-1}\left(E_{j_{2} \cdots j_{d}}\right)^{d / 2} \lesssim W_{1}^{d /\left(\ell_{1}+1\right)} \prod_{n=2}^{k}\left(\rho_{n}^{\left(d+\ell_{n}\right) / 2} W_{n}^{(d-1) /\left(\ell_{n}+1\right)}\right) .
$$

With (2.21) this will yield (2.18).

To give the details missing from the argument in the preceding paragraph we will need a lemma whose statement requires the introduction of some more notation: $\mathcal{A}_{d-1}$ will stand for the convex hull in $\mathbb{R}^{d-1}$ of the set of all permutations of the 
$(d-1)$-tuple $(1,2, \ldots, d-1)$. Recall that $\ell_{1}+\cdots+\ell_{k}+k-1=d-1$. If $\ell_{1}>0$, $\mathcal{A}_{d-1}^{\prime}$ is defined to be the collection of all permutations of $(d-1)$-tuples

$$
(d-1)(\frac{1}{\ell_{1}}\left(1, \ldots, \ell_{1}\right) ; \frac{1}{\ell_{2}+1}\left(1, \ldots, \ell_{2}\right) ; \ldots ; \frac{1}{\ell_{k}+1}\left(1, \ldots, \ell_{k}\right) ; \underbrace{\frac{1}{2}, \cdots, \frac{1}{2}}_{k-1 \text { times }}) .
$$

Note that if $k=1$, then $\mathcal{A}_{d-1}^{\prime}=\mathcal{A}_{d-1}$. For $k \geq 2$, define $\mathcal{A}_{d-1}^{\prime \prime}$ to be the collection of all permutations of $(d-1)$-tuples

$$
(d-1)(\frac{1}{\ell_{1}+1}\left(1, \ldots, \ell_{1}\right) ; \ldots ; \frac{1}{\ell_{k}+1}\left(1, \ldots, \ell_{k}\right) ; \underbrace{\frac{1}{2}, \cdots, \frac{1}{2}}_{k-2 \text { times }} ; 1) .
$$

(To simplify the notation, and since no confusion will result from doing so, we suppress the dependence of $\mathcal{A}_{d-1}^{\prime}$ and $\mathcal{A}_{d-1}^{\prime \prime}$ on $k$ and the $\ell_{n}$ 's.)

Lemma 2.2. The inclusions $\mathcal{A}_{d-1}^{\prime}, \mathcal{A}_{d-1}^{\prime \prime} \subset \mathcal{A}_{d-1}$ hold.

Moving towards (2.21), fix $n^{\prime} \in\{2, \ldots, k\}$. We will show that

$$
\begin{array}{r}
2^{m_{n^{\prime}}} \prod_{n=1}^{k} W_{n}^{(d-1) /\left(\ell_{n}+1\right)}\left(\prod_{\substack{1 \leq n \leq k \\
n \neq 1, n^{\prime}}} \rho_{n}^{(d-1) / 2}\right) \rho_{n^{\prime}}^{d-1} \\
\lesssim 2^{2\left[\left(\ell_{1}+1\right) m_{1}+\cdots+\left(\ell_{k}+1\right) m_{k}\right] /\left(d^{2}+d\right)} .
\end{array}
$$

Recall the definitions of the intervals $\left(\alpha_{i}, \beta_{i}\right)$, whose lengths $\Delta_{i}$ are the numbers $\rho_{n}(n=2, \ldots, k)$ along with the numbers $t_{i+1}-t_{i}$ for $i=p_{n-1}+1, \ldots, p_{n}-1$ and $n=1, \ldots, k$. Since

$$
W_{n}=\prod_{i=p_{n-1}+1}^{p_{n}-1}\left(t_{i+1}-t_{i}\right)^{e^{n}}
$$

for some choice of $\left\{e_{i}^{n}\right\}$ with

$$
\left\{e_{i}^{n}\right\}_{i=p_{n-1}+1}^{p_{n}-1}=\left\{1, \ldots, \ell_{n}\right\}
$$

it follows that

$$
\prod_{n=1}^{k} W_{n}^{(d-1) /\left(\ell_{n}+1\right)}\left(\prod_{\substack{1 \leq n \leq k \\ n \neq 1, n^{\prime}}} \rho_{n}^{(d-1) / 2}\right) \rho_{n^{\prime}}^{d-1}=\prod_{i=1}^{d-1} \Delta_{i}^{\sigma(i)},
$$

where the vector $\sigma=(\sigma(i))$ is in $\mathcal{A}_{d-1}^{\prime \prime}$ and where, if $i_{0}$ is the index for which $\left(\alpha_{i_{0}}, \beta_{i_{0}}\right)=\widetilde{J}_{n^{\prime}}$, then $\sigma\left(i_{0}\right)=d-1$ and so $\Delta_{i_{0}}^{\sigma\left(i_{0}\right)}=\rho_{n^{\prime}}^{d-1}$. By Lemma 2.2, the vector $\sigma$ is a convex combination

$$
\sigma=\sum_{q} \lambda_{q} \tau_{q}
$$

of vectors $\tau_{q}=\left(\tau_{q}(i)\right)$, each of which is a permutation of $(1, \ldots, d-1)$. Further, since $\sigma\left(i_{0}\right)=d-1$, we have $\tau_{q}\left(i_{0}\right)=d-1$ for each $q$. Now it follows from Lemma 2.1 that if the $c_{i}$ 's are nonnegative, then

$$
c_{i_{0}} \Delta_{i_{0}}^{d-1} \prod_{\substack{1 \leq i \leq d-1 \\ i \neq i_{0}}} \Delta_{i}^{\tau_{q}(i)} \lesssim \int_{t_{1}}^{t_{d}} \sum_{i=1}^{d-1} c_{i} \chi_{\left(\alpha_{i}, \beta_{i}\right)}(u) \psi\left(u ; t_{1}, \ldots, t_{d}\right) d u
$$


for each $q$. From (2.24), (2.25), and (2.26) it then follows that

$$
\begin{aligned}
& c_{i_{0}} \prod_{n=1}^{k} W_{n}^{(d-1) /\left(\ell_{n}+1\right)}\left(\prod_{\substack{1 \leq n \leq k \\
n \neq 1, n^{\prime}}} \rho_{n}^{(d-1) / 2}\right) \rho_{n^{\prime}}^{d-1} \\
& \lesssim \int_{t_{1}}^{t_{d}} \sum_{i=1}^{d-1} c_{i} \chi_{\left(\alpha_{i}, \beta_{i}\right)}(u) \psi\left(u ; t_{1}, \ldots, t_{d}\right) d u .
\end{aligned}
$$

Since (2.17) implies that $\omega \sim 2^{m_{n^{\prime}}}$ on $\widetilde{J}_{n^{\prime}}=\left(\alpha_{i_{0}}, \beta_{i_{0}}\right)$, we have

$$
\begin{aligned}
2^{m_{n^{\prime}}} & \prod_{n=1}^{k} W_{n}^{(d-1) /\left(\ell_{n}+1\right)}\left(\prod_{\substack{1 \leq n \leq k \\
n \neq 1, n^{\prime}}} \rho_{n}^{(d-1) / 2}\right) \rho_{n^{\prime}}^{d-1} \lesssim \int_{t_{1}}^{t_{d}} 2^{m_{n^{\prime}}} \chi_{\widetilde{J}_{n^{\prime}}}(u) \psi\left(u ; t_{1}, \ldots, t_{d}\right) d u \\
& \lesssim \int_{t_{1}}^{t_{d}} \omega(u) \psi\left(u ; t_{1}, \ldots, t_{d}\right) d u=J\left(t_{1}, \ldots, t_{d}\right) \lesssim 2^{2\left[\left(\ell_{1}+1\right) m_{1}+\cdots+\left(\ell_{k}+1\right) m_{k}\right] /\left(d^{2}+d\right)}
\end{aligned}
$$

by (2.3) and (2.19). This is (2.23). Analogous to (2.23) we will also need, in the case $\ell_{1}>0$, the estimate

$$
\begin{array}{r}
2^{m_{1}} W_{1}^{(d-1) / \ell_{1}} \prod_{n=2}^{k} W_{n}^{(d-1) /\left(\ell_{n}+1\right)} \prod_{n=2}^{k} \rho_{n}^{(d-1) / 2} \\
\lesssim 2^{2\left[\left(\ell_{1}+1\right) m_{1}+\cdots+\left(\ell_{k}+1\right) m_{k}\right] /\left(d^{2}+d\right)} .
\end{array}
$$

As before,

$$
W_{1}^{(d-1) / \ell_{1}} \prod_{n=2}^{k} W_{n}^{(d-1) /\left(\ell_{n}+1\right)} \prod_{n=2}^{k} \rho_{n}^{(d-1) / 2}=\prod_{i=1}^{d-1} \Delta_{i}^{\sigma(i)}
$$

where now $\sigma$ is in $\mathcal{A}_{d-1}^{\prime}$. With $\sigma=\sum_{q} \lambda_{q} \tau_{q}$ as in (2.25), Lemma 2.1 gives

$$
2^{m_{1}} \prod_{i=1}^{d-1} \Delta_{i}^{\tau_{q}(i)} \lesssim \int_{t_{1}}^{t_{d}} \sum_{1}^{d-1} 2^{m_{1}} \chi_{\left(\alpha_{i}, \beta_{i}\right)}(u) \psi\left(u ; t_{1}, \ldots, t_{d}\right) d u
$$

for each $q$. This leads, as before, to

$$
\begin{aligned}
2^{m_{1}} W_{1}^{(d-1) / \ell_{1}} \prod_{n=2}^{k} W_{n}^{(d-1) /\left(\ell_{n}+1\right)} \prod_{n=2}^{k} \rho_{n}^{(d-1) / 2} & \\
& \quad \lesssim \int_{t_{1}}^{t_{d}} \sum_{1}^{d-1} 2^{m_{1}} \chi_{\left(\alpha_{i}, \beta_{i}\right)}(u) \psi\left(u ; t_{1}, \ldots, t_{d}\right) d u .
\end{aligned}
$$

Since $\omega \gtrsim 2^{m_{1}}$ on $\left[t_{1}, t_{d}\right],(2.28)$ follows as in (2.27).

Now (2.21) will follow by considering a particular weighted geometric mean of the estimates (2.28) and (2.23). In fact, given the computations

$$
\begin{aligned}
& \frac{\ell_{1}}{d-1}+\frac{\ell_{2}+1}{d-1}+\frac{\ell_{3}+1}{d-1}+\cdots+\frac{\ell_{k}+1}{d-1}=1, \\
& \quad \frac{d-1}{\ell_{1}} \frac{\ell_{1}}{d-1}+\frac{d-1}{\ell_{1}+1}\left(\frac{\ell_{2}+1}{d-1}+\frac{\ell_{3}+1}{d-1}+\cdots+\frac{\ell_{k}+1}{d-1}\right)=\frac{d}{\ell_{1}+1},
\end{aligned}
$$

and

$$
(d-1) \frac{\ell_{n^{\prime}}+1}{d-1}+\frac{d-1}{2}\left(1-\frac{\ell_{n^{\prime}}+1}{d-1}\right)=\frac{d+\ell_{n^{\prime}}}{2}, \quad n^{\prime}=2, \ldots, k,
$$


(2.21) is an immediate consequence of (2.23) and (2.28).

Now the proof of (2.18) will be complete when we have explained how to choose $\left(t_{2}, \ldots, t_{d}\right) \in E_{j_{2} \ldots j_{d}}$ so that (2.22) holds. We will need another lemma and some more notation: recall that

$$
c_{1}<d_{1}<c_{2}<d_{2}<\cdots<c_{k}<d_{k} .
$$

Let $\delta_{n}=d_{n}-c_{n}$. Recall that $1 \leq p_{1}<p_{2}<\cdots<p_{k}=d, p_{0}=0, \ell_{n}=p_{n}-p_{n-1}-1$, and that

$$
c_{n} \leq t_{p_{n-1}+1}<\cdots<t_{p_{n}} \leq d_{n}
$$

for $n=1, \ldots k$. With $t_{p_{n-1}+1} \in\left[c_{n}, d_{n}\right)$, write $\mathbf{t}_{\mathbf{n}}$ for an $\ell_{n}$-tuple $\left(t_{p_{n-1}+2}, \ldots, t_{p_{n}}\right)$ satisfying (2.29) and $\mathbf{t}$ for the $\left(\ell_{1}+\cdots+\ell_{k}=d-k\right)$-tuple $\left(\mathbf{t}_{\mathbf{1}}, \ldots, \mathbf{t}_{\mathbf{k}}\right)$.

Lemma 2.3. The inequality

$$
m_{d-k}\left(\left\{\mathbf{t}: W_{1}^{d /\left(\ell_{1}+1\right)} \prod_{n=2}^{k} W_{n}^{(d-1) /\left(\ell_{n}+1\right)} \leq \mu\right\}\right) \lesssim \mu^{2 / d} \prod_{n=2}^{k} \delta_{n}^{\ell_{n} / d}
$$

holds for $\mu>0$.

Writing

$$
\left(t_{2}, \ldots, t_{d}\right)=\left(t_{p_{1}+1}, \ldots, t_{p_{k-1}+1} ; \mathbf{t}\right)
$$

with $\mathbf{t}$ as above, we use (2.29) to choose

$$
\left(t_{p_{1}+1}^{\prime}, \ldots, t_{p_{k-1}+1}^{\prime}\right) \in \prod_{n=2}^{k}\left[c_{n}, d_{n}\right]
$$

such that

$$
m_{d-k}\left(\left\{\mathbf{t}:\left(t_{p_{1}+1}^{\prime}, \ldots, t_{p_{k-1}+1}^{\prime} ; \mathbf{t}\right) \in E_{j_{2} \cdots j_{d}}\right\}\right) \geq \frac{m_{d-1}\left(E_{j_{2} \cdots j_{d}}\right)}{\prod_{n=2}^{k} \delta_{n}} .
$$

Let $c_{1}(d)>0$ be sufficiently small. Then if $\mu>0$ is such that

$$
\mu^{2 / d} \prod_{n=2}^{k} \delta_{n}^{\ell_{n} / d}=c_{1}(d) \frac{m_{d-1}\left(E_{j_{2} \cdots j_{d}}\right)}{\prod_{n=2}^{k} \delta_{n}},
$$

it follows from Lemma 2.3 that there is

$$
\left(t_{2}, \ldots, t_{d}\right) \in E_{j_{2} \cdots j_{d}}
$$

such that

$$
W_{1}^{d /\left(\ell_{1}+1\right)} \prod_{n=2}^{k} W_{n}^{(d-1) /\left(\ell_{n}+1\right)}>\mu=c_{2}(d) \frac{m_{d-1}\left(E_{j_{2} \cdots j_{d}}\right)^{d / 2}}{\prod_{n=2}^{k} \delta_{n}^{\left(d+\ell_{n}\right) / 2}} .
$$

Recalling that $\rho_{n}$ is the length of $\tilde{J}_{n}$, so that $\delta_{n}=d_{n}-c_{n} \lesssim \rho_{n}$ by (2.16) and (2.14), (2.22) follows. 


\section{Proofs of Lemmas}

Proof of Lemma 2.1. The proof is by induction on $d$. Since $\psi\left(u ; t_{1}, t_{2}\right)=\chi_{\left[t_{1}, t_{2}\right]}(u)$, the case $d=2$ is clear. Fix $p \in\{1, \ldots, d-1\}$ and then a choice of $\left\{e_{i}\right\}_{i \neq p}$ so that $\left\{e_{i}\right\}_{i \neq p}=\{1, \ldots, d-2\}$. Let $q$ satisfy $e_{q}=1$. We will give the argument in the case $q<p$, the case $q>p$ being similar. Let $m_{i}$ be the midpoint of $\left[\alpha_{i}, \beta_{i}\right]$. Define intervals $I_{1}, \ldots I_{d-1}$ and $J_{1}, \ldots J_{d-1}$ as follows:

$$
\begin{gathered}
I_{j}=\left(\alpha_{i}, m_{i}\right), J_{i}=\left(m_{i}, \beta_{i}\right) \text { if } i<q, \\
I_{q}=\left(\alpha_{q}, m_{q}\right), J_{q}=\emptyset, \\
I_{i}=\left(m_{i}, \beta_{i}\right), J_{i}=\left(\alpha_{i}, m_{i}\right) \text { if } i>q .
\end{gathered}
$$

We use the identity

$$
\psi\left(u ; t_{1}, \ldots, t_{d}\right)=\int_{t_{1}}^{t_{2}} \cdots \int_{t_{d-1}}^{t_{d}} \psi\left(u ; s_{1}, \ldots, s_{d-1}\right) d s_{1} \cdots d s_{d-1},
$$

a consequence of the proof of Lemma 2.3 in [2], and the fact that $\psi\left(u, s_{1}, \ldots, s_{d-1}\right)$ is supported on $\left[s_{1}, s_{d-1}\right]$ to write

$$
\begin{aligned}
\int_{t_{1}}^{t_{d}} f(u) \psi\left(u ; t_{1}, \ldots, t_{d}\right) d u & \\
& =\int_{t_{1}}^{t_{2}} \cdots \int_{t_{d-1}}^{t_{d}} \int_{s_{1}}^{s_{d-1}} f(u) \psi\left(u ; s_{1}, \ldots, s_{d-1}\right) d u d s_{1} \cdots d s_{d-1}
\end{aligned}
$$

Then

$$
\text { (3.1) } \geq \int_{I_{1}} \cdots \int_{I_{d-1}} \int_{s_{1}}^{s_{d-1}} \sum_{\substack{1 \leq i \leq d-1 \\ i \neq q}} \nu_{i} \chi_{J_{i}}(u) \psi\left(u ; s_{1}, \ldots, s_{d-1}\right) d u d s_{1} \cdots d s_{d-1} \text {. }
$$

Now if $s_{i} \in I_{i}$ for $i=1, \ldots, d-1$, then $J_{i} \subset\left(s_{i}, s_{i+1}\right)$ if $i<q$ and $J_{i} \subset\left(s_{i-1}, s_{i}\right)$ if $i>q$. Thus, assuming the lemma for $d-1$ and noting that $\left\{e_{i}-1\right\}_{i \neq p, q}=$ $\{1, \ldots, d-3\}$, it follows that

$$
\int_{s_{1}}^{s_{d-1}} \sum_{\substack{1 \leq i \leq d-1 \\ i \neq q}} \nu_{i} \chi_{J_{i}}(u) \psi\left(u ; s_{1}, \ldots, s_{d-1}\right) d u \gtrsim \nu_{p} \Delta_{p}^{d-2} \prod_{\substack{1 \leq i \leq d-1 \\ i \neq p, q}} \Delta_{i}^{e_{i}-1}
$$

and so

$$
\text { (3.1) } \gtrsim \nu_{p}\left(\prod_{i=1}^{d-1} \Delta_{i}\right) \Delta_{p}^{d-2} \prod_{\substack{1 \leq i \leq d-1 \\ i \neq p, q}} \Delta_{i}^{e_{i}-1}=\nu_{p} \Delta_{p}^{d-1} \prod_{\substack{1 \leq i \leq d-1 \\ i \neq p}} \Delta_{i}^{e_{i}}
$$

completing the proof of Lemma 2.1

Proof of Lemma 2.2. Lemma 2.2 is the statement that if

$$
\ell_{1}+\cdots+\ell_{k}+k-1=r
$$

then (a) if $\ell_{1}>0, k \geq 2$, and $\mathcal{A}_{r}^{\prime}$ is the collection of all permutations of $r$-tuples

$$
r(\frac{1}{\ell_{1}}\left(1, \ldots, \ell_{1}\right) ; \frac{1}{\ell_{2}+1}\left(1, \ldots, \ell_{2}\right) ; \ldots ; \frac{1}{\ell_{k}+1}\left(1, \ldots, \ell_{k}\right) ; \underbrace{\frac{1}{2}, \cdots, \frac{1}{2}}_{k-1 \text { times }}),
$$


we have $\mathcal{A}_{r}^{\prime} \subset \mathcal{A}_{r}$, and (b) if $k \geq 2$ and $\mathcal{A}_{r}^{\prime \prime}$ is the collection of all permutations of $r$-tuples

$$
r(\frac{1}{\ell_{1}+1}\left(1, \ldots, \ell_{1}\right) ; \ldots ; \frac{1}{\ell_{k}+1}\left(1, \ldots, \ell_{k}\right) ; \underbrace{\frac{1}{2}, \cdots, \frac{1}{2}}_{k-2 \text { times }} ; 1),
$$

we have $\mathcal{A}_{r}^{\prime \prime} \subset \mathcal{A}_{r}$. We will show these inclusions by induction on $k$. We require the following two facts, which we establish at the end of the proof of this lemma:

$$
\left(\frac{1}{\ell_{k-1}+1}\left(1, \ldots, \ell_{k-1}\right) ; \frac{1}{\ell_{k}+1}\left(1, \ldots, \ell_{k}\right)\right) \in\left(\frac{1}{\ell_{k-1}+\ell_{k}+1}\right) \mathcal{A}_{\ell_{k-1}+\ell_{k}}
$$

and

$$
\left(\frac{1}{\ell_{k-1}+1}\left(1, \ldots, \ell_{k-1}\right) ; \frac{1}{\ell_{k}+1}\left(1, \ldots, \ell_{k}\right) ; \frac{1}{2}\right) \in\left(\frac{1}{\ell_{k-1}+\ell_{k}+2}\right) \mathcal{A}_{\ell_{k-1}+\ell_{k}+1} \text {. }
$$

If $k=2$ and $\ell_{1}>1$, a vector (3.2) can be written as

$$
r\left(\frac{1}{\ell_{1}}\left(1, \ldots, \ell_{1}-1\right) ; 1 ; \frac{1}{\ell_{2}+1}\left(1, \ldots, \ell_{2}\right) ; \frac{1}{2}\right)
$$

and therefore, by (3.4), as a linear combination of permutations of vectors

$$
r\left(\frac{1}{\ell_{1}+\ell_{2}+1}\left(1, \ldots, \ell_{1}+\ell_{2}\right) ; 1\right) \in \mathcal{A}_{r} .
$$

And if $k=2$ and $\ell_{1}=1$, then (3.2) can be written as

$$
r\left(1 ; \frac{1}{\ell_{2}+1}\left(1, \ldots, \ell_{2}\right) ; \frac{1}{1+1}(1)\right)
$$

and therefore, by (3.3), as a linear combination of permutations of the vector

$$
r\left(1 ; \frac{1}{\ell_{2}+2}\left(1, \ldots, \ell_{2}+1\right)\right) \in \mathcal{A}_{r} .
$$

Thus (a) holds for $k=2$. The fact that (b) holds for $k=2$ follows similarly from (3.3). So assume that $k \geq 3$ and that (a) and (b) hold with $k-1$ in place of $k$.

To show that (b) holds for $k$, fix a vector

$$
r(\frac{1}{\ell_{1}+1}\left(1, \ldots, \ell_{1}\right) ; \ldots ; \frac{1}{\ell_{k-1}+1}\left(1, \ldots, \ell_{k-1}\right) ; \frac{1}{\ell_{k}+1}\left(1, \ldots, \ell_{k}\right) ; \underbrace{\frac{1}{2}, \cdots, \frac{1}{2}}_{k-2 \text { times }} ; 1)
$$

in $\mathcal{A}_{r}^{\prime \prime}$. It follows from (3.4) that (3.5) can be written as a convex combination of permutations of vectors

$$
\begin{aligned}
r\left(\frac{1}{\ell_{1}+1}\left(1, \ldots, \ell_{1}\right) ; \ldots\right. & ; \frac{1}{\ell_{k-2}+1}\left(1, \ldots, \ell_{k-2}\right) \\
& \frac{1}{\ell_{k-1}+\ell_{k}+2}\left(1, \ldots, \ell_{k-1}+\ell_{k}+1\right) ; \underbrace{\frac{1}{2}, \cdots, \frac{1}{2}}_{k-3 \text { times }} ; 1),
\end{aligned}
$$

and our induction assumption implies that each permutation of (3.6) is in $\mathcal{A}_{r}$. This establishes (b) for $k$. 
To see that (a) holds for $k$, note that the argument above shows that a vector

$$
r(\frac{1}{\ell_{1}}\left(1, \ldots, \ell_{1}\right) ; \ldots ; \frac{1}{\ell_{k-1}+1}\left(1, \ldots, \ell_{k-1}\right) ; \frac{1}{\ell_{k}+1}\left(1, \ldots, \ell_{k}\right) ; \underbrace{\frac{1}{2}, \cdots, \frac{1}{2}}_{k-1 \text { times }})
$$

of the form (3.2) can be written as a convex combination of permutations of vectors

$$
\begin{aligned}
r\left(\frac{1}{\ell_{1}}\left(1, \ldots, \ell_{1}\right) ; \ldots ;\right. & \frac{1}{\ell_{k-2}+1}\left(1, \ldots, \ell_{k-2}\right) ; \\
& \frac{1}{\ell_{k-1}+\ell_{k}+2}\left(1, \ldots, \ell_{k-1}+\ell_{k}+1\right) ; \underbrace{\frac{1}{2}, \cdots, \frac{1}{2}}_{k-2 \text { times }})
\end{aligned}
$$

and so, by the induction assumption, is in $\mathcal{A}_{r}$.

It remains to establish (3.3) and (3.4). We require an alternate description of $\mathcal{A}_{r}$. Let $\mathcal{A}_{r}^{*}$ be the set

$$
\left\{\left(a_{1}, \ldots, a_{r}\right): \sum_{j=1}^{r} a_{j}=\frac{r(r+1)}{2} \text { and } \sum_{j \in \mathcal{E}} a_{j} \geq \frac{|\mathcal{E}|(|\mathcal{E}|+1)}{2} \text { if } \mathcal{E} \subset\{1, \ldots, r\}\right\} .
$$

We want to show that $\mathcal{A}_{r}=\mathcal{A}_{r}^{*}$, and it is enough to show that each extreme point of the convex set $\mathcal{A}_{r}^{*}$ is a permutation of $(1, \ldots, r)$. So assume that $\left(a_{1}, \ldots, a_{r}\right)$ is an extreme point of $\mathcal{A}_{r}^{*}$. Without loss of generality we may also assume $a_{1} \leq a_{2} \leq$ $\cdots \leq a_{r}$

Our first step will be to show that

$$
a_{1}<a_{2}<\cdots<a_{d} .
$$

To this end, assume that $a_{s}=a_{s+1}$ for some $s \in\{1, \ldots, d-1\}$. We will show that if either $s \in \mathcal{E}$ and $s+1 \notin \mathcal{E}$ or $s+1 \in \mathcal{E}$ and $s \notin \mathcal{E}$, then

$$
\sum_{j \in \mathcal{E}} a_{j}>\frac{|\mathcal{E}|(|\mathcal{E}|+1)}{2} \text {. }
$$

If (3.8) holds, it will follow that there is $\delta>0$ such that the vector obtained from $\left(a_{1}, \ldots, a_{r}\right)$ by replacing $a_{s}$ and $a_{s+1}$ by $a_{s}+\eta$ and $a_{s+1}-\eta$ is in $\mathcal{A}_{r}$ whenever $|\eta|<\delta$. This implies that $\left(a_{1}, \ldots, a_{r}\right)$ is not extreme. To show (3.8) we begin with an observation:

$$
\text { if } a_{k}=a_{k+1} \text {, then } \sum_{j=1}^{k} a_{j}>\frac{k(k+1)}{2} \text {. }
$$

(To see (3.9), observe that the assumption $\sum_{j=1}^{k} a_{j}=\frac{k(k+1)}{2}$ and the inequality $\sum_{j=1}^{k+1} a_{j} \geq \frac{(k+1)(k+2)}{2}$ together imply that $a_{k+1} \geq k+1$ and so $a_{k} \geq k+1$ if $a_{k}=a_{k+1}$. Then

$$
\sum_{j=1}^{k} a_{j}=a_{k}+\sum_{j=1}^{k-1} a_{j} \geq(k+1)+\frac{(k-1) k}{2},
$$

contradicting $\sum_{j=1}^{k} a_{j}=\frac{k(k+1)}{2}$.) Returning to (3.8), we will write $k=|\mathcal{E}|$ and consider three cases: 
Case $I(k<s)$ : Here we will show that if $s \in \mathcal{E}$ or $s+1 \in \mathcal{E}$, then (3.8) holds. Assuming $s \in \mathcal{E}$, it follows that

$$
\frac{k(k+1)}{2} \leq \sum_{j=1}^{k} a_{j}=\sum_{j=1}^{k-1} a_{j}+a_{k} \leq \sum_{j=1}^{k-1} a_{j}+a_{s} \leq \sum_{j \in \mathcal{E}} a_{j} .
$$

Thus if (3.8) fails, then $a_{k}=a_{k+1}$ (since $a_{k}=a_{s}$ ) and $\sum_{j=1}^{k} a_{j}=k(k+1) / 2$, contradicting (3.9). The case $s+1 \in \mathcal{E}$ is similar.

Case II $(k=s)$ : We will just observe that if $a_{s}=a_{s+1}$, then (3.8) holds. In fact, since

$$
\sum_{j \in \mathcal{E}} a_{j} \geq \sum_{j=1}^{k} a_{j}
$$

this follows immediately from (3.9).

Case III $(k>s)$ : We will show that if either $s \notin \mathcal{E}$ or $s+1 \notin \mathcal{E}$, then (3.8) holds. Write $\mathcal{E}=\left\{j_{1}, j_{2}, \ldots, j_{k}\right\}$ with $j_{1}<\cdots<j_{k}$. Since $k>s$ and either $s \notin \mathcal{E}$ or $s+1 \notin \mathcal{E}$, it follows that $j_{k}>k$. Then, since $1 \leq j_{1}, \ldots, k \leq j_{k}$, the inequality

$$
\frac{k(k+1)}{2} \leq \sum_{l=1}^{k} a_{l} \leq \sum_{l=1}^{k} a_{j_{l}}=\sum_{j \in \mathcal{E}} a_{j}
$$

shows that if (3.8) fails, then $a_{k}=a_{j_{k}}$ and $\sum_{j=1}^{k} a_{j}=k(k+1) / 2$, again resulting in a contradiction of (3.9). Thus (3.8), and so (3.7), are established.

Now suppose that $\left(a_{1}, \ldots, a_{r}\right)$ is extreme and (3.7) holds. If $a_{j} \geq j$ for $j=$ $1, \ldots, r$, then the condition

$$
\sum_{j=1}^{r} a_{j}=r(r+1) / 2
$$

forces $\left(a_{1}, \ldots, a_{r}\right)=(1, \ldots, r)$. But if we have $a_{j}<j$ for any $j$, we can choose $t$ such that $a_{t}<t$ and $a_{j} \geq j$ for $j=1, \ldots, t-1$ (the condition $a_{1} \geq 1$ implies that $t>1)$. Since

$$
\sum_{j=1}^{t} a_{j} \geq t(t+1) / 2
$$

we can choose $s$ with $s \leq t-1, a_{s}>s$, and $a_{j}=j$ if $s<j<t$. Thus

$$
\left(a_{1}, \ldots, a_{r}\right)=\left(a_{1}, \ldots, a_{s-1}, a_{s}, s+1, \ldots, t-1, a_{t}, a_{t+1}, \ldots, a_{r}\right) .
$$

It follows from (3.10) and $a_{t}<t$ that

$$
\sum_{j=1}^{p} a_{j}>p(p+1) / 2
$$

for $p=s, \cdots, t-1$. Thus there is $\delta>0$ such that if $|\eta|<\delta$, then

$$
\left(a_{1}, \ldots, a_{s-1}, a_{s}+\eta, s+1, \ldots, t-1, a_{t}-\eta, a_{t+1}, \ldots, a_{r}\right) \in \mathcal{A}_{r}^{*},
$$

where we have used the fact that (3.7) implies that the entries of the vector in (3.11) are nondecreasing if $\delta$ is small enough. Then $\left(a_{1}, \ldots, a_{r}\right)$ cannot be an extreme point of $\mathcal{A}_{r}^{*}$. Thus $a_{j} \geq j$ for $j=1, \ldots, r$ and so $\left(a_{1}, \ldots, a_{r}\right)=(1, \ldots, r)$ as desired. 
We return to the proofs for (3.3) and (3.4). Since $\mathcal{A}_{\ell_{k-1}+\ell_{k}}=\mathcal{A}_{\ell_{k-1}+\ell_{k}}^{*}$, (3.3) will follow from checking that if $m \leq \ell_{k-1}$ and $n \leq \ell_{k}$, then

$$
\frac{\ell_{k-1}+\ell_{k}+1}{\ell_{k-1}+1} \frac{m(m+1)}{2}+\frac{\ell_{k-1}+\ell_{k}+1}{\ell_{k}+1} \frac{n(n+1)}{2} \geq \frac{(n+m)(n+m+1)}{2} .
$$

This inequality is equivalent to the inequality

$$
m(m+1) \ell_{k}\left(\ell_{k}+1\right)+n(n+1) \ell_{k-1}\left(\ell_{k-1}+1\right) \geq 2 m n\left(\ell_{k-1}+1\right)\left(\ell_{k}+1\right) .
$$

And the easily checked inequality

$$
\sqrt{m(m+1) n(n+1) \ell_{k-1}\left(\ell_{k-1}+1\right) \ell_{k}\left(\ell_{k}+1\right)} \geq m n\left(\ell_{k-1}+1\right)\left(\ell_{k}+1\right)
$$

shows that (3.13) follows from the inequality between arithmetic and geometric means.

Similarly, to show that (3.4) holds it is enough to check that if $m \leq \ell_{k-1}$ and $n \leq \ell_{k}$, then the inequalities

$$
\frac{\ell_{k-1}+\ell_{k}+2}{\ell_{k-1}+1} \frac{m(m+1)}{2}+\frac{\ell_{k-1}+\ell_{k}+2}{\ell_{k}+1} \frac{n(n+1)}{2} \geq \frac{(n+m)(n+m+1)}{2}
$$

and

$$
\begin{aligned}
& \frac{\ell_{k-1}+\ell_{k}+2}{\ell_{k-1}+1} \frac{m(m+1)}{2}+\frac{\ell_{k-1}+\ell_{k}+2}{\ell_{k}+1} \frac{n(n+1)}{2}+\frac{\ell_{k-1}+\ell_{k}+2}{2} \\
& \geq \frac{(n+m+1)(n+m+2)}{2}
\end{aligned}
$$

hold. Since (3.14) follows from (3.12), it is enough to establish (3.15). Now inequality (3.12) is equivalent to

$$
\frac{m(m+1)}{2\left(\ell_{k-1}+1\right)}+\frac{n(n+1)}{2\left(\ell_{k}+1\right)} \geq \frac{(n+m)(n+m+1)}{2\left(\ell_{k-1}+\ell_{k}+1\right)},
$$

while (3.15) is equivalent to

$$
\begin{aligned}
\frac{m(m+1)}{2\left(\ell_{k-1}+1\right)}+\frac{n(n+1)}{2\left(\ell_{k}+1\right)}+\frac{1}{2} & \geq \frac{(n+m+1)(n+m+2)}{2\left(\ell_{k-1}+\ell_{k}+2\right)} \\
& =\left(\frac{(n+m)(n+m+1)}{2}+(n+m+1)\right) \\
& \times\left(\frac{1}{\ell_{k-1}+\ell_{k}+1}-\frac{1}{\left(\ell_{k-1}+\ell_{k}+1\right)\left(\ell_{k-1}+\ell_{k}+2\right)}\right) .
\end{aligned}
$$

Thus it is enough to show that

$$
\begin{aligned}
\frac{1}{2}+\frac{(n+m)(n+m+1)}{2\left(\ell_{k-1}+\ell_{k}+1\right)\left(\ell_{k-1}+\ell_{k}+2\right)}+\frac{n+m+1}{\left(\ell_{k-1}+\ell_{k}+1\right)\left(\ell_{k-1}+\ell_{k}+2\right)} & \\
& \geq \frac{n+m+1}{\ell_{k-1}+\ell_{k}+1} .
\end{aligned}
$$

This is equivalent to the inequality

$$
\left(\ell_{k-1}+\ell_{k}+1\right)+\frac{(n+m+1)(n+m+2)}{\ell_{k-1}+\ell_{k}+2} \geq 2(n+m+1),
$$

which follows from the arithmetic-geometric mean inequality and the easily checked

$$
(n+m+1)\left(\ell_{k-1}+\ell_{k}+2\right) \leq\left(\ell_{k-1}+\ell_{k}+1\right)(n+m+2),
$$

itself a consequence of $m \leq \ell_{k-1}, n \leq \ell_{k}$. 
Proof of Lemma 2.3. Recall that $c_{n} \leq t_{p_{n-1}+1}<\cdots<t_{p_{n}} \leq d_{n}$ and that $W_{n}=$ $W\left(t_{p_{n-1}+1}, \ldots, t_{p_{n}}\right)$. It follows that $W_{n} \lesssim \delta_{n}^{\ell_{n}\left(\ell_{n}+1\right) / 2}$ and so

$$
\begin{aligned}
& m_{d-k}\left(\left\{\mathbf{t}: W_{1}^{d /\left(\ell_{1}+1\right)} \prod_{n=2}^{k} W_{n}^{(d-1) /\left(\ell_{n}+1\right)} \leq \mu\right\}\right) \\
& \lesssim \sum_{2^{p_{2}} \leq \delta_{2}^{\ell_{2}\left(\ell_{2}+1\right) / 2}} \cdots \sum_{2^{p_{k}} \leq \delta_{k}^{\ell_{k}\left(\ell_{k}+1\right) / 2}} \prod_{n=2}^{k}\left|\left\{W_{n} \leq 2^{p_{n}}\right\}\right| \\
& \cdot\left|\left\{W_{1} \leq\left(\mu / \prod_{n=2}^{k} 2^{p_{n}(d-1) /\left(\ell_{n}+1\right)}\right)^{\left(\ell_{1}+1\right) / d}\right\}\right|,
\end{aligned}
$$

where

$$
\left|\left\{W_{n} \leq \lambda\right\}\right|=m_{\ell_{n}}\left(\left\{\left(t_{p_{n-1}+2}, \ldots, t_{p_{n}}\right): W\left(t_{p_{n-1}+1}, t_{p_{n-1}+2}, \ldots, t_{p_{n}}\right) \leq \lambda\right\}\right) .
$$

We will need the estimate

$$
\left|\left\{W_{n} \leq \lambda\right\}\right| \lesssim \lambda^{2 /\left(\ell_{n}+1\right)}
$$

To show (3.17) and, more generally, to show that

$$
m_{p}\left(\left\{\left(s_{1}, \ldots, s_{p}\right): 0 \leq s_{1} \leq \cdots \leq s_{p}, W\left(0, s_{1}, \ldots, s_{p}\right) \leq \lambda\right\}\right) \leq C(p) \lambda^{2 /(p+1)},
$$

we will argue by induction on $p$. (Inequality (3.18) is an analog of a result, Proposition 2.4 (i) in [1, from [5] and [6].) The case $p=1$ is clear, so assume that (3.18) holds. If we make the change of variables

$$
u_{1}=s_{1}, u_{2}=s_{2}-s_{1}, \ldots, u_{p}=s_{p}-s_{p-1},
$$

then

$$
\begin{aligned}
& m_{p}\left(\left\{\left(s_{1}, \ldots, s_{p}\right): 0 \leq s_{1} \leq \cdots \leq s_{p}, W\left(0, s_{1}, \ldots, s_{p}\right) \leq \lambda\right\}\right) \\
& =p ! m_{p}\left(\left\{\left(u_{1}, \ldots, u_{p}\right): 0 \leq u_{1} \leq \cdots \leq u_{p}, \prod_{j=1}^{p}\left(u_{j}\right)^{j} \leq \lambda\right\}\right) .
\end{aligned}
$$

Now

$$
\begin{gathered}
m_{p+1}\left(\left\{\left(u_{1}, \ldots, u_{p+1}\right): 0 \leq u_{1} \leq \cdots \leq u_{p+1}, \prod_{j=1}^{p+1}\left(u_{j}\right)^{j} \leq \lambda\right\}\right) \\
=\int_{0}^{\infty} m_{p}\left(\left\{\left(u_{1}, \ldots, u_{p}\right): 0 \leq u_{1} \leq \cdots \leq u_{p} \leq u_{p+1},\right.\right. \\
\left.\left.\prod_{j=1}^{p} u_{j}^{j} \leq \lambda /\left(u_{p+1}\right)^{p+1}\right\}\right) d u_{p+1} .
\end{gathered}
$$

Thus the estimate

$$
\begin{gathered}
m_{p}\left(\left\{\left(u_{1}, \ldots, u_{p}\right): 0 \leq u_{1} \leq \cdots \leq u_{p+1}, \prod_{j=1}^{p}\left(u_{j}\right)^{j} \leq \lambda /\left(u_{p+1}\right)^{p+1}\right\}\right) \\
\leq C(p) \min \left\{\left(u_{p+1}\right)^{p},\left(\lambda /\left(u_{p+1}\right)^{p+1}\right)^{2 /(p+1)}\right\}
\end{gathered}
$$

a consequence of (3.18), shows that (3.18) holds with $p$ replaced by $p+1$. 
Now, using (3.17) and some algebra, we have

$$
\left(\underline{3.16)} \lesssim \mu^{2 / d} \sum_{2^{p_{2}} \leq \delta_{2}^{\ell_{2}\left(\ell_{2}+1\right) / 2}} \ldots \sum_{2^{p_{k}} \leq \delta_{k}^{\ell_{k}\left(\ell_{k}+1\right) / 2}} \prod_{n=2}^{k} 2^{2 p_{n} /\left[d\left(\ell_{n}+1\right]\right)} \lesssim \mu^{2 / d} \prod_{n=2}^{k} \delta_{n}^{\ell_{n} / d} .\right.
$$

This gives the desired conclusion and therefore completes the proof of Lemma 2.3.

\section{ACKNOWLEDGment}

The author would like to express his gratitude to Jong-Guk Bak and Andreas Seeger: this note is a byproduct of the collaboration that led to [1] and [2].

\section{REFERENCES}

[1] J.-G. Bak, D. Oberlin and A. Seeger, Restriction of Fourier transforms to curves and related oscillatory integrals, Amer. J. Math. 131 (2009), 277-311. MR.2503984

[2] - Restriction of Fourier transforms to curves. II: some classes with vanishing torsion, J. Australian Math. Soc. 85 (2008), 1-28. MR2460861

[3] M. Christ, On the restriction of the Fourier transform to curves: endpoint results and the degenerate case, Trans. Amer. Math. Soc. 287 (1985), 223-238. MR766216 (87b:42018)

[4] S. Dendrinos and J. Wright, Fourier restriction to polynomial curves I: a geometric inequality, preprint.

[5] S.W. Drury and B. Marshall, Fourier restriction theorems for curves with affine and Euclidean arclengths, Math. Proc. Cambridge Philos. Soc. 97 (1985), 111-125. MR764500 (87b:42019)

[6] _ Fourier restriction theorems for degenerate curves, Math. Proc. Cambridge Philos. Soc. 101 (1987), 541-553. MR 878901 (88e:42026)

[7] D. Oberlin, Fourier restriction for affine arclength measures in the plane, Proc. Amer. Math. Soc. 129 (2001), 3303-3305. MR1845006 (2002g:42013)

[8] Some convolution inequalities and their applications, Trans. Amer. Math. Soc. 354 (2002), 2541-2556. MR 1885663 (2002j:42016)

[9] P. Sjölin, Fourier multipliers and estimates of the Fourier transform of measures carried by smooth curves in $R^{2}$, Studia Math. 51 (1974), 169-182. MR0385437(52:6299)

Department of Mathematics, Florida State University, Tallahassee, Florida 32306

E-mail address: oberlin@math.fsu.edu 